\title{
$P$ wave dispersion in patients with erectile dysfunction
}

\author{
CAN RAMAZAN ÖNCEL ${ }^{1, *}$, ALI AKKOÇ ${ }^{2}$ \\ ${ }^{1}$ Department of Cardiology, School of Medicine, Alanya Alaaddin Keykubat University, Alanya, Turkey \\ ${ }^{2}$ Department of Urology, School of Medicine, Alanya Alaaddin Keykubat University, Alanya, Turkey \\ *Corresponding author: Can Ramazan Öncel; Department of Cardiology, School of Medicine, Alanya Alaaddin Keykubat University, Konya \\ Çimento Street No: 80, Alanya 07450, Antalya, Turkey; Phone: +90 50637151 99; Fax: +90 242510 61 39; E-mail: r_oncel@hotmail.com
}

(Received: March 3, 2019; Accepted: April 12, 2019)

\begin{abstract}
Background and aims: $\mathrm{P}$ wave dispersion (PWD) has been reported to be a non-invasive electrocardiographic predictor for atrial fibrillation. The aim of this study is to evaluate PWD between men with erectile dysfunction (ED) and healthy controls in order to investigate whether PWD was prolonged in patients with ED and related to severity of the disease. Methods: This study included a total of 72 men (42 patients with ED and 30 healthy controls). Demographic data and clinical features were recorded on admission. An electrocardiographic evaluation was obtained to measure PWD values for both patients and controls. Results: Maximum P wave duration was $108.5 \pm 4.7$ and $108.3 \pm 4.3$ in ED group and control group, respectively $(p=0.748)$. Minumum $\mathrm{P}$ wave duration was significantly higher in the control group than in the ED group. PWD was $48.1 \pm 5.9$ in the ED group. As a result, PWD was prolonged in patients with $\mathrm{ED}(48.1 \pm 5.9$ vs. $38.0 \pm 3.9, p<0.05)$. A significant negative correlation was observed between IIEF score and PWD values $(p<0.05, r=-0.662)$. Conclusions: Patients with ED exhibited prolonged PWD values compared with normal controls. In addition, PWD was found to be associated with severity of the disease.
\end{abstract}

Keywords: erectile dysfunction, P wave dispersion, autonomic nervous system, endothelial dysfunction, electrocardiography

\section{Introduction}

Erectile dysfunction (ED) is defined as the loss of ability to achieve or maintain a satisfactory erection long enough to engage in sexual intercorse. It is a worldspread problem, which mainly affects men older than 40 years old and its frequency increases in the older people [1]. As ED has many organic and non-organic factors, ED and cardiovascular diseases (CVDs) have common risk factors, such as hypertension, diabetes mellitus, dyslipidemia, obesity, metabolic syndrome, smoking, and sedanter lifestyle. In addition to these modifiable and unmodifiable risk factors, ED and CVD share two common pathophysiological mechanisms: autonomic nervous system (ANS) dysfunction and endothelial dysfunction $[2,3]$.

Deterioration of autonomic function can lead to cardiovascular events, which can increase morbidity and mortality. Moreover, it can lead to ED and the relationship between ED and ANS has been evaluated in previous studies. Another important mechanism underlying ED is endothelial dysfunction, which disrupts corpora cavernosal smooth muscle relaxation $[4,5]$.

Potential pathophysiological mechanisms including inflammation, oxidative stress, endothelial dysfunction, ANS dysfunction, hemodynamic, and vascular changes are the main causes and consequences of cardiovascular disorders and ED. Atrial fibrillation (most common sustained arrhythmia) is also associated with vascular dysfunction and its inflammation [6].

$\mathrm{P}$ wave dispersion (PWD) is a non-invasive electrocardiographic marker for atrial remodeling and a strong predictor of atrial arrhythmias and especially atrial fibrillation. P wave duration and dispersion have been reported to be influenced by the autonomic tone and reactive oxygen species. It has also been shown that a prolonged PWD was associated with coronary artery disease and prediction of atrial fibrillation in patients with acute coronary syndrome. This suggests that PWD could also be influenced by endothelial dysfunction [7-9].

This is an open-access article distributed under the terms of the Creative Commons Attribution-NonCommercial 4.0 International License, which permits unrestricted use, distribution, and reproduction in any medium for non-commercial purposes, provided the original author and source are credited, a link to the CC License is provided, and changes - if any - are indicated. 
$\mathrm{P}$ wave parameters in ED have not yet been investigated. In this study, we aimed to evaluate PWD in ED in order to evaluate cardiac autonomic dysfunction, endothelial dysfunction, and potential risk of developing atrial fibrillation in patients with ED.

\section{Materials and Methods}

The study population consisted of 42 patients with ED who were followed up at urology outpatient clinic. The control group consisted of 30 healthy controls.

The International Index of Erectile Function Questionnaire (IIEF-5) was used to evaluate the erectile status of patients. The questionnaire includes five questions and each question is scored between 1 and 5 points to find out a global sexual function score. According to the results, a total score of $<22$ was defined as ED [10]. Demographic data and clinical features were recorded on admission. Blood pressures were measured and blood samples were taken for hematological and biochemical parameters after an overnight 12 -h fasting. Patients under 18 years and over 70 years of age were excluded. Patients with known CVD, congenital heart disease, metabolic-endocrine disease, renal disease, hypo-hyper thyroidism, diabetes mellitus, hypertension, acute-chronic respiratory diseases, neurological/ psychiatric disorders, rheumatologic entities, gastrointestinal diseases, use of drugs that can cause ED or affect PWD, systemic infection, and electrocardiography (ECG) without clearly analyzable $\mathrm{P}$ wave were excluded from the study. The local ethics committee approved the study protocol.

\section{Electrocardiography (ECG)}

A 12-lead surface ECG during sinus rhythm was obtained from each participant in supine position following $10-\mathrm{min}$ rest with $10 \mathrm{~mm} / \mathrm{mV}$ amplitude and $25 \mathrm{~mm} / \mathrm{s}$ paper speed. All of the ECG results were scanned and transferred to a personal computer for PWD calculation, as manual measurement has less accuracy compared with a computerized digital system. The point of first detectable atrial deflection from the isoelectric line was defined as the onset of the $\mathrm{P}$ wave. Return to the isoelectric line was defined as the end of the $P$ wave. $P$ wave duration was measured in all 12 leads on the surface ECG in order to evaluate maximum and minimum $\mathrm{P}$ wave duration $(\mathrm{P} \max$ and $\mathrm{P}$ min, respectively). The difference calculated by substracting $\mathrm{P}$ min from $P$ max was defined as PWD. All ECG measurements were performed by an independent cardiologist who was blinded to the subjects' clinical status.

\section{Echocardiography}

Transthoracic echocardiographic examinations were performed in all participants at the left lateral decubitus position to evaluate conventional parameters. All echocardiographic measurements were taken according to the guidelines of American Society of Echocardiography.

\section{Statistical analysis}

SPSS 22.0 (SPSS Inc., Chicago, IL, USA) program was used in analysis. The data were presented as mean \pm standard deviation. The Kolmogorov-Smirnov test was used to examine if variables are normally distributed. Comparison between the two groups was performed with independent sample $t$-test and Mann-Whitney $U$ test. Spearman's test was used for correlation analysis between IIEF score and PWD values. Intraobserver and interobserver agreements were assessed using the intraclass correlation analysis. The $p$ value less than 0.05 was considered statistically significant.

\section{Results}

Forty-two patients with ED and 30 controls without ED were included in the study. The demographic and clinical characteristics of the patients and healthy controls are shown in Table $I$.

There were no significant differences between the patients and the controls in terms of age distribution. Body mass index and body surface area were similiar in the two groups. Furthermore, no significant differences were observed in systolic and diastolic blood pressures or in heart rates between the two groups.

Echocardiographic and electrocardiographic characteristics of the study groups were shown in Table II. Left ventricular ejection fraction, left ventricular end-diastolic diameter, left ventricular end-systolic diameter, left atrial (LA) diameter, interventricular septum, posterior wall thickness, and mitral inflow parameters were similiar in both groups.

Maximum $P$ wave duration was $108.5 \pm 4.7$ and $108.3 \pm 4.3$ in ED group and control group, respectively $(p=0.748)$. Minumum $\mathrm{P}$ wave duration was significantly shorter in ED group as compared to that in the control group. PWD was $48.1 \pm 5.9$ in ED group and $38.0 \pm 3.9$ in the control group. As a result, PWD was prolonged in patients with $\mathrm{ED}(p<0.05)$.

A significant negative correlation was observed between IIEF score and PWD values $(p<0.05, r=-0.662)$. In PWD measurements, intraobserver and interobserver agreement results showed a good reproducibility and small variability $(r=0.95,95 \% \mathrm{CI}=0.878-0.98$ and $r=0.946$, $95 \%$ CI $=0.869-0.978$, respectively).

\section{Discussion}

In this study, we found that patients with ED have longer PWD values than those with healthy controls. To the best of our knowledge, this is the first study to evaluate the impact of ED on $\mathrm{P}$ wave duration. 
Table I Demographic, clinical, and echocardiographic variables of study group

\begin{tabular}{|c|c|c|c|}
\hline & Control $(n=30)$ & Erectile dysfunction $(n=42)$ & $p$ \\
\hline \multicolumn{4}{|c|}{ Demographic and clinical variables } \\
\hline Age (years) & $38.0 \pm 9.5$ & $39.9 \pm 6.7$ & 0.333 \\
\hline $\operatorname{BMI}\left(\mathrm{kg} / \mathrm{m}^{2}\right)$ & $26.9 \pm 4.4$ & $24.8 \pm 4.4$ & 0.053 \\
\hline Total cholesterol & $182.7 \pm 45.9$ & $171.7 \pm 31.6$ & 0.500 \\
\hline TG & $167.2 \pm 68.7$ & $174.9 \pm 97.3$ & 0.950 \\
\hline LDL & $110.7 \pm 35.3$ & $114.8 \pm 31.9$ & 0.694 \\
\hline Glucose & $87.8 \pm 9.0$ & $86.7 \pm 9.4$ & 0.485 \\
\hline Creatinin & $0.8 \pm 0.1$ & $0.79 \pm 0.1$ & 0.895 \\
\hline $\mathrm{Hb}$ & $15.3 \pm 0.8$ & $14.8 \pm 1.4$ & 0.062 \\
\hline SBP & $118.8 \pm 8.5$ & $120.5 \pm 10.8$ & 0.488 \\
\hline DBP & $73.8 \pm 6.7$ & $74.2 \pm 3.2$ & 0.958 \\
\hline $\mathrm{HR}$ & $73.1 \pm 8.6$ & $76.5 \pm 9.4$ & 0.100 \\
\hline IIEF score & - & $15.3 \pm 2.9$ & - \\
\hline \multicolumn{4}{|c|}{ Echocardiographic variables } \\
\hline LVDD (cm) & $46.8 \pm 4.2$ & $48.3 \pm 3.6$ & 0.153 \\
\hline $\operatorname{LVSD}(\mathrm{cm})$ & $28.3 \pm 3.9$ & $28.9 \pm 3.2$ & 0.370 \\
\hline $\mathrm{EF}(\%)$ & $70.3 \pm 5.8$ & $69.8 \pm 6.2$ & 0.583 \\
\hline IVS & $9.5 \pm 0.9$ & $9.7 \pm 1.0$ & 0.297 \\
\hline PW & $9.4 \pm 1.1$ & $9.2 \pm 1.2$ & 0.420 \\
\hline $\operatorname{Lad}(\mathrm{cm})$ & $34.1 \pm 3.1$ & $33.2 \pm 2.2$ & 0.145 \\
\hline $\mathrm{E} / \mathrm{A}$ ratio & $1.2 \pm 0.3$ & $1.1 \pm 0.3$ & 0.293 \\
\hline
\end{tabular}

Data are presented as mean \pm standard deviation values. TG: triglyceride; BMI: body mass index; LDL: low-density lipoprotein; Hb: hemoglobin; SBP: systolic blood pressure; DBP: diastolic blood pressure; HR: heart rate; IIEF: International Index of Erectile Function Questionnaire; Lad: anterior-posterior left atrial diameter; EF: ejection fraction; LVDD: left ventricular diastolic diameter; LVSD: left ventricular systolic diameter; IVS: interventricular septum; PW: posterior wall; E/A: early to late atrial mitral Doppler peak flow velocity

Table II Electrocardiographic variables of study group

\begin{tabular}{lcrr} 
Electrocardiographic variables & Control $(n=30)$ & Erectile dysfunction $(n=42)$ & $p$ \\
P maximum & $108.3 \pm 4.3$ & $108.5 \pm 4.7$ & 0.748 \\
P minimum & $70.2 \pm 3.3$ & $60.5 \pm 5.0$ & $<0.050$ \\
PWD & $38.0 \pm 3.9$ & $48.1 \pm 5.9$ & $<0.050$ \\
\hline
\end{tabular}

Data are presented as mean \pm standard deviation values. PWD: P wave dispersion

It has been demonstrated that ED was associated with an increased risk of CVDs [11-13]. As ED and subclinical CVD have similiar risk factors, such as age, obesity, smoking, and hypertension. They also have common pathophysiological mechanisms including endothelial dysfunction and autonomic dysfunction [14, 15].

Endothelial dysfunction leads to insufficient relaxation of the vascular smooth muscle of the corpus cavernosum and it is one of the important mechanisms underlying ED. ED can be considered as an early manifestation of endothelial dysfunction and precursor or predictor of other forms of heart disease including atrial fibrillation. Endothelial impairment has been described in patients with atrial fibrillation previously $[16,17]$. Especially any condition that impairs endothelial function could cause ED [18]. Lin et al. [19] investigated the association between atrial fibrillation and ED and they found that incidence of ED was significantly lower in patients without AF than those with AF. Yllmaz et al. demonstrated the relationship between ED and paroxysmal lone atrial fibrillation. They concluded that endothelial dysfunction as a consequence of atrial fibrillation could be the cause of ED in their study population [20].

Another important mechanism underlying ED is autonomic dysfunction, which plays a major role in cardiovascular mortality and morbidity [21-23]. An imbalance between parasympathetic and sympathetic system has been demonstrated in patients with ED. Decreased 
parasympathetic activity and increased catecholaminergic state lead to ED $[24,25]$. The normal functioning of the ANS is important for both cardiovascular system and erectile function. It has been demonstrated that the ANS has participated in the initiation of idiopathic atrial fibrillation [26]. Furthermore, it has been shown that autonomic remodeling leads to increased atrial sympathetic innervation in atrial fibrillation patients [27].

Kucukdurmaz et al. [28] reported that cardiac autonomic functions are impaired in patients with ED. Chen et al. [29] showed that patients with ED had significant cardiac sympathetic hyperactivity and cardiac vagal impairment. Autonomic impairment may change the atrial conduction velocity and affect the duration of the $\mathrm{P}$ wave [30]. The accuracy of PWD measurement, especially minumum $\mathrm{P}$ wave duration, could be influenced by the low resolution of standard 12-lead ECG due to vector differences between the leads. Due to this reason, we measured $\mathrm{P}$ wave duration using computerized digital system. In addition, it has been concluded that increased PWD is usually associated with increased maximum $\mathrm{P}$ wave duration. However, in this study, we found that minimum $\mathrm{P}$ wave was shorter and PWD was significantly longer in patients with ED as compared to those in the control group. It is revealed that increased sympathetic activity causes vasoconstriction and loss of erection. It has also been reported that increased sympathetic tone shortens PWD in normal subjects [31]. Similar to our results, Tukek at al. [32] demonstrated that shorter minimum $\mathrm{P}$ wave duration was associated with paroxysmal atrial fibrillation in patients with increased LA diameter. Dilaveris et al. [33] reported shorter $P$ wave duration as an independent predictor of common atrial fibrillation. In an another study, shorter minimum $\mathrm{P}$ wave duration was found to be an important predictor of atrial fibrillation for patients undergoing coronary artery bypass grafting [34]. PWD is a valuable non-invasive method for predicting patients who have potential risk of developing atrial fibrillation. It has been reported that interatrial conduction delays are associated with initiating and maintaining atrial fibrillation $[35,36]$. Furthermore, some authors reported increased PWD is associated with stable coronary artery disease, carotid atherosclerosis, and slow coronary phenomenon $[9,37,38]$. This suggests that another pathophysiological mechanism for increased PWD is endothelial dysfunction as well as autonomic imbalance. Endothelial dysfunction, oxidative stress, atherosclerosis, and autonomic dysfunction are the main causes and simultaneously consequences of atrial fibrillation.

Platek et al. [39] reported that ED was present in more than half of the study group in their cross-sectional, epidemiological study conducted in patients with a primary diagnosis of atrial fibrillation. We believed that impairment in autonomic cardiac function (increased conduction velocity from heightened sympathetic drive and shorter minimum $\mathrm{P}$ wave duration) with endothelial dysfunction could be the cause of prolonged PWD in patients with ED. Age, left atrial size, left ventricular ejection fraction, and left ventricular diastolic function parameters could be related to prolonged PWD in different patient groups. However, we did not found any difference between our study groups in terms of these parameters.

The limitations of this study are as follows:

1. This was a single-center study and a relatively small number of patients were included.

2. We did not evaluate the association between PWD and duration of ED. In addition, we could not make a correlation analysis between serum testosterone levels and electrocardiographic parameters in patients with ED and control subjects. We also did not investigate other evidence of autonomic dysfunction and examine its relationship with PWD to provide more mechanistic insight.

3. Furthermore, the psychosocial factors (e.g., anxiety, stress, enviromental factors during measurement, and mental state) of ED were not evaluated.

4. The results of this study cannot be applied to general population.

In conclusion, prolonged PWD may be associated with impaired autonomic and endothelial function in patients with ED. PWD analysis can be used as a non-invasive method to predict the risk of developing atrial fibrillation in ED patients. The findings of our analysis can guide for the further clinical practice. However, these findings must be confirmed by further large scale and prospective studies to support our hypothesis.

Funding sources: The authors received no financial support for the research, authorship, and/or publication of this article.

Authors' contribution: CRÖ is responsible for patient selection, analysis, and writing of the manuscript. AA contributed to the data collection and editing of the manuscript.

Conflict of interest: The authors declared no potential conflict of interest with respect to the research, authorship, and/or publication of this article.

Data availability: The data sets generated and/or analyzed during this study are available from the corresponding author on reasonable request.

\section{References}

1. Echeverri Tirado LC, Ferrer JE, Herrera AM: Aging and erectile dysfunction. Sex Med Rev 4, 63-73 (2016)

2. Araujo AB, Hall SA, Ganz P, Chiu GR, Rosen RC, Kupelian V, Travison TG, McKinlay JB: Does erectile dysfunction contribute to cardiovascular disease risk prediction beyond the Framingham risk score? J Am Coll Cardiol 55, 350-356 (2010)

3. Pop-Busui R, Hotaling J, Braffett BH, Cleary PA, Dunn RL, Martin CL, Jacobson AM, Wessells H, Sarma AV, DCCT/EDIC Research 
Group: Cardiovascular autonomic neuropathy, erectile dysfunction and lower urinary tract symptoms in men with type 1 diabetes: Findings from the DCCT/EDIC. J Urol 193, 2045-2051 (2015)

4. Jung J, Jo HW, Kwon H, Jeong NY: Clinical neuroanatomy and neurotransmitter-mediated regulation of penile erection. Int Neurourol J 18, 58-62 (2014)

5. Leoni LA, Fukushima AR, Rocha LY, Maifrino LB, Rodrigues B: Physical activity on endothelial and erectile dysfunction: A literature review. Aging Male 17, 125-130 (2014)

6. Takahashi N, Ishibashi Y, Shimada T, Sakane T, Ohata S, Sugamori T, Ohta Y, Inoue S, Nakamura K, Shimizu H, Katoh H, Sano K, Murakami Y, Hashimoto M: Atrial fibrillation impairs endothelial function of forearm vessels in humans. J Card Fail 7, 45-54 (2001)

7. Conlon R, Tanner R, David S, Szeplaki G, Galvin J, Keaney J, Keelan E, Boles U: Evaluation of the Tp-Te interval, QTc and P wave dispersion in patients with coronary artery ectasia. Cardiol Res 8, 280-285 (2017)

8. Aytemir K, Ozer N, Atalar E, Sade E, Aksöyek S, Ovünç K, Oto A, Ozmen F, Kes S: P wave dispersion on 12-lead electrocardiography in patients with paroxysmal atrial fibrillation. Pacing Clin Electrophysiol 23, 1109-1112 (2000)

9. Rosiak M, Bolinska $\mathrm{H}$, Ruta J: $\mathrm{P}$ wave dispersion and $\mathrm{P}$ wave duration on SAECG in predicting atrial fibrillation in patients with acute myocardial infarction. Ann Noninvasive Electrocardiol 7, 363-368 (2002)

10. Rhoden EL, Telöken C, Sogari PR, Vargas Souto CA: The use of the simplified International Index of Erectile Function (IIEF-5) as a diagnostic tool to study the prevalence of erectile dysfunction. Int J Impot Res 14, 245-250 (2002)

11. Hackett G, Kirby M, Wylie K, Heald A, Ossei-Gerning N, Edwards $\mathrm{D}$, Muneer A: British society for sexual medicine guidelines on the management of erectile dysfunction in Men-2017. J Sex Med 15, $430-457$ (2018)

12. Dong JY, Zhang YH, Qin LQ: Erectile dysfunction and risk of cardiovascular disease: Meta-analysis of prospective cohort studies. J Am Coll Cardiol 58, 1378-1385 (2011)

13. Gandaglia G, Briganti A, Jackson G, Kloner RA, Montorsi F, Montorsi P, Vlachopoulos C: A systematic review of the association between erectile dysfunction and cardiovascular disease. Eur Urol 65, 968-978 (2014)

14. Osondu CU, Vo B, Oni ET, Blaha MJ, Veledar E, Feldman T, Agatston AS, Nasir K, Aneni EC: The relationship of erectile dysfunction and subclinical cardiovascular disease: A systematic review and meta analysis. Vasc Med 23, 9-20 (2018)

15. Lee JY, Joo KJ, Kim JT, Cho ST, Cho DS, Won YY, Choi JB: Heart rate variability in men with erectile dysfunction. Int Neurourol J 15, 87-91 (2011)

16. Kirby M, Jackson G, Betteridge J, Friedli K: Is erectile dysfunction a marker for cardiovascular disease? Int J Clin Pract 55, 614-618 (2001)

17. Krishnamoorthy S, Lim SH, Lip G: Assessment of endothelial (dys) function in atrial fibrillation. Ann Med 41, 576-590 (2009)

18. McMahon CG: Erectile dysfunction. Intern Med J 44, 18-26 (2014)

19. Lin WY, Lin CS, Lin CL, Cheng SM, Lin WS, Kao CH: Atrial fibrillation is associated with increased risk of erectile dysfunction: A nationwide population-based cohort study. Int J Cardiol 190, 106-110 (2015)

20. Yılmaz S, Kuyumcu MS, Akboga MK, Sen F, Balcı KG, Balcı MM, Özeke Ö, Aras D, Aydoğdu S: The relationship between erectile dysfunction and paroxysmal lone atrial fibrillation. J Interv Card Electrophysiol 46, 245-251 (2016)

21. Ulucan Ş, Kaya Z, Keser A, Katlandur H, Karanfil M, Ateş İ: Deterioration of heart rate recovery index in patients with erectile dysfunction. Anatol J Cardiol 16, 264-269 (2016)
22. Cole CR, Blackstone EH, Pashkow FJ, Snader CE, Lauer MS: Heart-rate recovery immediately after exercise as a predictor of mortality. N Engl J Med 341, 1351-1357 (1999)

23. Lauer MS, Okin PM, Larson MG, Evans JC, Levy D: Impaired heart rate response to graded exercise. Prognostic implications of chronotropic incompetence in the Framingham Heart Study. Circulation 93, 1520-1526 (1996)

24. Cheitlin MD: Sexual activity and cardiac risk. Am J Cardiol 96, 24M-28M (2005)

25. Giuliano F, Rampin O: Neural control of erection. Physiol Behav 83, 189-201 (2004)

26. Chen PS, Chen LS, Fishbein MC, Lin SF, Nattel S: Role of the autonomic nervous system in atrial fibrillation: Pathophysiology and therapy. Circ Res 114, 1500-1515 (2014)

27. Gould PA, Yii M, McLean C, Finch S, Marshall T, Lambert GW, Kaye DM: Evidence for increased atrial sympathetic innervation in persistent human atrial fibrillation. Pacing Clin Electrophysiol 29, 821-829 (2006)

28. Kucukdurmaz F, Acar G, Resim S: Deterioration of chronotropic responses and heart rate recovery indices in men with erectile dysfunction. Sex Med 6, 8-14 (2018)

29. Chen CJ, Kuo TB, Tseng YJ, Yang CC: Combined cardiac sympathetic excitation and vagal impairment in patients with nonorganic erectile dysfunction. Clin Neurophysiol 120, 348-352 (2009)

30. Cheema AN, Ahmed MW, Kadish AH, Goldberger JJ: Effects of autonomic stimulation and blockade on signal-averaged $\mathrm{P}$ wave duration. J Am Coll Cardiol 26, 497-502 (1995)

31. Chang IC, Austin E, Krishnan B, Benditt DG, Quay CN, Ling LH, Chen LY: Shorter minimum p-wave duration is associated with paroxysmal lone atrial fibrillation. J Electrocardiol 47, 106-112 (2014)

32. Tukek T, Akkaya V, Atilgan D, Demirel E, Ozcan M, Güven O, Korkut F: Effect of left atrial size and function on P-wave dispersion: A study in patients with paroxysmal atrial fibrillation. Clin Cardiol 24, 676-680 (2001)

33. Dilaveris PE, Gialafos EJ, Chrissos D, Andrikopoulos GK, Richter DJ, Lazaki E, Gialafos JE: Detection of hypertensive patients at risk for paroxysmal atrial fibrillation during sinus rhythm by computerassisted P wave analysis. J Hypertens 17, 1463-1470 (1999)

34. Hashemi Jazi M, Amirpour A, Zavvar R, Behjati M, Gharipour M: Predictive value of $\mathrm{P}$ wave duration and dispersion in post coronary artery bypass surgery atrial fibrillation. ARYA Atherosclerosis 8 , 59-62 (2012)

35. Shimizu A, Centurion OA: Electrophysiological properties of the human atrium in atrial fibrillation. Cardiovasc Res 54, 302-314 (2002)

36. Centurión OA: Clinical implications of the $\mathrm{P}$ wave duration and dispersion: Relationship between atrial conduction defects and abnormally prolonged and fractionated atrial endocardial electrograms. Int J Cardiol 134, 6-8 (2009)

37. Akin F, Firatli I, Katkat F, Gurmen T, Ayca B, Kalyoncuoglu M, Abaci O, Sari M, Ersanli M, Kucukoglu S, Yigit Z: P-wave dispersion and its relationship with the severity of the disease in patients with stable coronary artery disease. North Clin Istanb 1, 65-70 (2014)

38. Ozuğuz U, Ergün G, Işık S, Gökay F, Tütüncü $Y$, Akbaba G, Berker D, Güler S: Association between C-reactive protein, carotid intima-media thickness and P-wave dispersion in obese premenopausal women: An observational study. Anatol J Cardiol 12, 40-46 (2012)

39. Platek AE, Hrynkiewicz-Szymanska A, Kotkowski M, Szymanski FM, Syska-Suminska J, Puchalski B, Filipiak KJ: Prevalence of erectile dysfunction in atrial fibrillation patients: A cross-sectional, epidemiological study. Pacing Clin Electrophysiol 39, 28-35 (2016) 